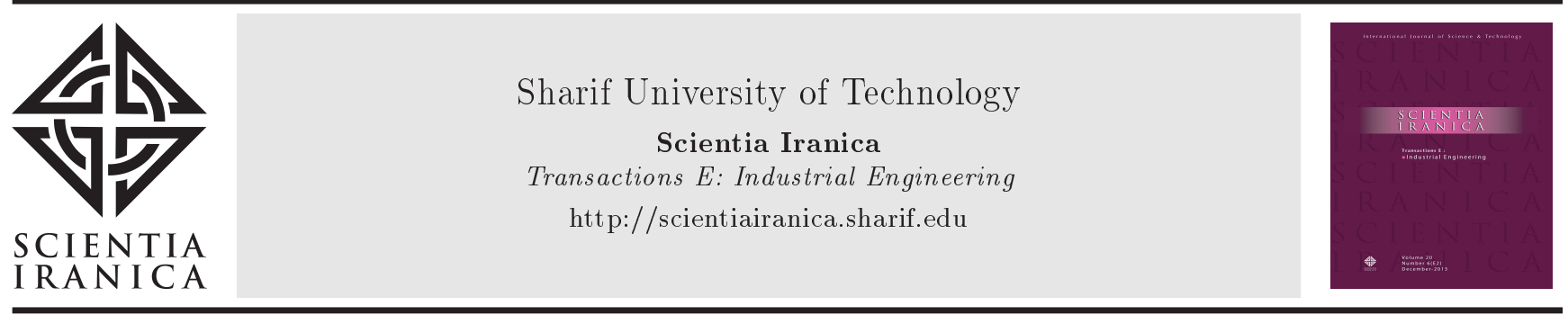

\title{
An architectural solution for virtual computer integrated manufacturing systems using ISO standards
}

\author{
J. Delaram and O. Fatahi Valilai* \\ Department of Industrial Engineering, Advanced Manufacturing Laboratory, Sharif University of Technology, Tehran, Iran.
}

Received 11 March 2017; received in revised form 7 May 2018; accepted 4 August 2018

KEYWORDS
CIM (Computer
Integrated
Manufacturing);
VCIM (Virtual
Computer Integrated
Manufacturing);
Manufacturing system
architecture;
Axiomatic Design
(AD) theory;
ISO standards.

\section{Introduction}

Very recently and following the fourth industrial revolution (Industry 4.0), manufacturing environments have been faced with the paradigm of globalization. This paradigm urges new necessities for manufacturing environments such as concentration on customer demands, emphasis on increasing quality and its continuous improvement, and the shortening of the products' lifecycle [1-4]. Researchers applied Information Tech-

\footnotetext{
*. Corresponding author. Tel.: +982166165706; Fax: +982166022702

E-mail addresses: jalal.delaram@ie.sharif.edu (J. Delaram); FValilai@sharif.edu (O. Fatahi Valilai)
}

doi: $10.24200 /$ sci. 2018.20799 nology (IT) to solve these challenges [4-11]. Computer Integrated Manufacturing (CIM) is considered as a research field to develop effective IT solutions. The CIM solutions have produced incredible revolutions and enhancements in manufacturing and production systems [11-16]. There are many pieces of related research [16-20] that emphasize the application of CIMbased solutions to realize integrated manufacturing systems [20-22].

Despite the enormous number of studies directed at global manufacturing paradigms, the proposed solutions can be grouped as traditional CIM solutions. These solutions have deficiencies and, thus, cannot fulfill the whole requirements of globalized manufacturing environments [22-24]. Among such deficiencies, the following problems can be named: dominant lacks, inadequate equipment and information, distance barri- 
ers, scheduling and resource allocation, facility sharing problems and communication obstacle, and the absence of an efficient way to handle lifecycle issues $[7,25,26]$. This motivates the proposition of new ideas and solutions for a flexible and comprehensive integrating methodology to overcome the aforementioned lacks. Many studies have recommended the Virtual CIM (VCIM) paradigm as one of the most effective methodologies for today's globalization paradigm $[14,20,27,28]$. VCIM is known to be an evolvement of computer integrated manufacturing solutions, which has been extended from the traditional CIM solutions to fulfill a global integrated manufacturing system beyond the traditional and localized boundaries [27-29]. Of all applicable and challenging areas for CIM/VCIM systems, its architecture is known to be more challenging. Architecture is the backbone of a system and defines how the system is implemented with respect to its goals associated with different aspects such as functional, informational, organizational, etc.

The concept of the CIM/VCIM systems is approached by two types of systems: Extended Enterprise (EE) and Virtual Enterprise (VE). EEs are composed of a single core as the head and many Large Enterprises (LEs) in their supply chain. LEs and other parts together create a tightly coupled structure that provides input service or product for a central factory. Usually, the central factory is interpreted as the main company or EE, and the other LEs are interpreted as the first-tier and second-tier suppliers. The central factory performs assembly functions on the manufactured items, which have been manufactured by the LEs, like Boeing and IBM [30,31]. The VEs are those enterprises that are composed of many Small to Medium Enterprises (SMEs) connected to each other via computer networks. The VEs [32,33] are often joined together to share skills and resources for responding better to business opportunities. The structure of today's market for outsourcing and benefiting from serviceoriented environments has encouraged the spread of the virtual environment paradigm and elaboration of its mechanisms [34-37]; the SMEs enjoy great characteristics to adapt and grow in such environments. SMEs are characterized to be distributed across the globe [8], and a VE is a manifestation of the distributed collaborative network [38]. Two of the major characteristics of VEs are regarded as loosely coupled and temporary, meaning that SMEs can register in a network and, then, accept to perform a defined task. They can unregister only after completing their assumed tasks; thus, the presence and absence of each SME do not disrupt the VE. As an example of a VE, Federated Laboratories of the United States Army (FedLabs) establish a close partnership among Army Research Laboratories and several industrial and academic organizations $[39,40]$.

These two types of enterprises strive for invest- ment in innovative IT-based solutions such as VCIM for a VE and CIM for an EE [14,41], and the need for an architectural solution for them is a key concern. System Architecture is the master plan based on which one can organize and structure a VE or an $\mathrm{EE}$ and perceive its policies, strategies, mechanisms, and processes for the exchange of information, goods, services, and money or preparation of hardware and infrastructures [42].

According to dispersion and diversity features of VEs, each SME can utilize different computer integrated systems. A VCIM with the capability of enabling a network of interconnected global CIM systems can establish real-time connections and collaboration in an effective manner among its components $[16,29]$. The dispersion dimension of a VE is also found in EEs due to their large size. Besides, an EE has many subordinates that can be considered as SMEs for a VE. Therefore, there are many reasons that a CIM for an EE can be interpreted as a VCIM for a VE [43]. In addition, many common drawbacks in a similar situation exist. Many research studies have been conducted in the area of VCIM solutions for VEs or CIM solutions for EEs; however, there is still a considerable gap regarding an effective solution $[13,14,20]$. In the following sections, the paper will elaborate on these gaps and attempt to tackle them with an architectural solution.

\section{Literature review}

Considering the discussed five-layer approach, valuable methodologies for supporting the VCIM systems have been developed in many pieces of research. However, concentrating on a five-layer approach, the studies have experienced drawbacks that prevent them from enabling an efficient and reliable global integrated system for manufacturing firms.

Zhang et al. [44] proposed a solution using CORBA (Common Object Request Broker Architecture) that consists of three layers. Through these layers, the hardware aspects of the manufacturing system are considered at the infrastructure level at the bottom of their architecture. With another view, Huang et al. [45] proposed a holonic framework for virtual enterprises. This framework has a hierarchical and heterarchical structure for controlling the system. In the proposed framework, six types of holons are considered. Resource holons are exploited to represent a resource in the real world such as machines, computers, persons, etc. The objective is to cover all hardware aspects in a manufacturing system. In addition, Huang's architecture includes two main holons named as (a) Virtual Enterprise Holon (VEH) and (b) Member Enterprise Holon (MEH). A VEH is responsible for managing and handling the registered $\mathrm{MEH}$. The MEH 
involves subholons such as Task Holon (TH), Planning Holon $(\mathrm{PH})$, etc. The other type of holon in the architecture is the holon for scheduling functions in a manufacturing system.

Wang et al. [13], Nagalingam and Lin [12], and Zhou [20] proposed an agent-based approach to develop an architectural solution for CIM systems. In this solution, the agents are classified into three levels: (a) Customer Agents (CA), (b) Facilitator Agents (FA), and (c) Resource Agents (RA). The CAs stand as an interface for interoperating with outer environments; FAs are responsible for managing the received requests and decomposing them. In addition, RAs are designed for considering functions required for the system such as designing, inspection, manufacturing, etc. Within each of FAs and RAs, KBs (Knowledge Bases) and DBs (Data Bases) are regarded. The KB supplies knowledge for RAs and develops strategies for manufacturing organizations. Moreover, the DB provides informational requirements, such as delivery time and total cost, for the product of a manufacturing system.

Odrey and Mejí [46] proposed an architectural solution composed of three layers: production layer, mediator layer, and recovery layer. These layers have a hierarchal structure and address scheduling and planning issues in manufacturing systems. The solution is equipped with recovery agents and control agents. The recovery agents represent diagnostic and error recovery or generate recovery schedules. The control agents stand for performing the delegated tasks and creating an ultimate execution plan. Further to that, there is a planner agent that is responsible for all processes of scheduling functions in the manufacturing systems.

Hernandez-Matias [47] developed an architectural solution that mainly covers the informational aspects. It includes a reference model for managing the information and a data warehouse for saving the information. The framework of the paper is exploited from a hierarchical point of view to develop the reference information model. In the reference model, the level of an activity is considered for functions in an enterprise to classify, manage, and allocate them.

Lin and Jeng [48] developed a framework including an information layer, a control layer, and a function layer. They have considered the entire functions of a manufacturing system through an SEMATECH CIM framework. The information layer provides standard data and information for the other layers of the system. The control layer monitors the manufacturing functions of the system. The control has been augmented using Manufacturing Execution System (MES), and it also covers MRP (Manufacturing Resource Planning), CAD (Computer Aided Design), and CAM (Computer Aided Manufacturing) systems. The functional layer stands for designing, planning, and manufacturing the application of the manufacturing system.
Nahm and Ishikawa [49] developed a Multi Agentbased Architecture (MAA) that is highly focused on inter-enterprise functions such as integration and collaboration. They defined hybrid agents in MAA to cover the entire behavior of the system. Hybrid Behavior Agent (HBA) and Hybrid Interaction Agent (HIA) are presented in this purpose. HBA covers all discrete and continuous behavior, and HIA involves the communication among the agents in the system. Further, they implicitly consider data and information flow through defining the interaction types between HBAs and HIAs.

\section{Five-layer approach}

In this section, this study proposes a layered architecture to define different aspects of the solution and provide a review of the studied researches. This layered architecture elaborates on different aspects of a CIM system based on five layers, as discussed in detail in previous studies [21,50-52]. The fivelayer architecture enables a wider look to consider a CIM system. Each layer focuses on an operational aspect of a manufacturing system. The first layer, or physical layer, considers aspects related to the hardware, equipment, and human workers. These aspects transform input resources into a product by direct physical resource processing. The second layer, or function layer, describes the dynamic behavior of a manufacturing system accomplished through product development processes. The function layer produces a model that includes process flowcharts coupled with physical layer. The third layer, or organizational layer, focuses on organizational and management structures of the system. All human and non-human characteristics within the organizational roles and configurations are defined in this layer. The fourth layer, or information Layer, manages the data and informational aspects of a computer integrated system. These aspects can include data and information requirements. The fifth layer, or control layer, considers the whole supportive processes such as monitoring and control, inspection, and decision-making. This layer monitors the whole system processes in the aforementioned layers and covers indirect support and supervision, which are necessary in manufacturing systems.

\section{Axiomatic design theory}

Suh created Axiomatic Design (AD) in the mid-1970s and, then, published the idea in "The Principle of Design" [53]. The name of the "axiomatic" is inspired by the utilization of design principles or design axioms that govern the process of design. The AD application spectrum is very wide and applicable to many fields [5461]. Generally, the process of design misses a formal 
approach [61,62], and $\mathrm{AD}$ supports the designers and developers to formalize the process of design $[63,64]$. In the axiomatic-based design process, motivation is considered as a customer requirement. A number of functional requirements can be found in relation to the customer requirement. Then, designers seek to find the parameters of the model and specify an appropriate solution. Therefore, AD stipulates that the design process has four general domains: customer domain, functional domain, physical domain, and process domain [65-67]. In a sequential order, starting from customer domain, the Customer Attributes (CAs) should be defined and, then, the CAs should be mapped to the functional domain and translated into Functional Requirements (FRs). Then, the FRs should be mapped to the Design Parameters (DPs) in physical domain, known as design process. Finally, the DPs should be satisfied with some Process Values (PVs) to address the implementation aspects $[68,69]$. Figure 1 illustrates this process.

The sequential process of AD guides the designer from "what we want to achieve" to "how we achieve it" [69]. AD uses two axioms in this process: Independence axiom and information axiom. The satisfaction level of these axioms determines the goodness of the design. The independence axiom maintains the independency of FRs to show that the FRs are defined as the minimum set of independent requirements that characterizes the design goals through functional domain. In addition, the information axiom minimizes the information content of the design, and shows that the best design among many other designs that satisfy the independence axiom is the one with the smallest information content [70-72]. If FRs are assumed as vectors with $m$ component and DPs as vectors with $n$ component, there is a $m \times n$ matrix to connect these two in a matrix representation:

$$
[F R]_{m}=[A]_{m \times n}[D P]_{n} .
$$

The matrix $A$ is interpreted as a design matrix and used for quality assessment of the design. Thus, based on the structure of the matrix $A$, the following alternatives are made possible:
- Uncoupled design:

$$
\forall(m . n) \in N . m \neq n: A_{m n}=0,
$$

Eq. (2) represents a diagonal matrix and reveals a one-to-one mapping between every member in the sets of FRs and DPs. In AD theory, this state is interpreted as each FR is contingent on one and only one DP. This case is the best possible one $[73,74]$.

- Decoupled design:

$$
\begin{aligned}
& \left(\forall(m . n) \in N . m>n: A_{m n}=0\right) \text { or } \\
& \left(\forall(m . n) \in N . m<n: A_{m n}=0\right) .
\end{aligned}
$$

Eq. (3) represents a triangular matrix in which all elements on one side of the diagonal of the matrix $A$ are nil, which shows a one-to-many mapping between members of the sets of FRs and DPs. In the AD theory, this state is interpreted since some of FRs are dependent on more than one DP. There are some considerations to benefit from this situation as a decouple design situation $[54,75]$.

- Coupled design:

$$
\begin{aligned}
& \exists\left(m_{1} \cdot n_{1}\right) \cdot\left(m_{2} \cdot n_{2}\right) \in N \cdot\left(m_{1}>n_{1}\right) \text { and } \\
& \left(m_{2}<n_{2}\right): A_{m_{1} n_{1}} \neq 0 \text { and } A_{m_{2} n_{2}} \neq 0 .
\end{aligned}
$$

This matrix shows a many-to-many mapping between members of the sets of FRs and DPs. In the $\mathrm{AD}$ theory, this state is interpreted since each DP affects not only the FR but also other functional requirements, and it is itself affected by them. This case is the worst possible one $[76,77]$.

\section{The architectural solution}

\subsection{Defining the first-level FR/DP}

Based on AD zigzag approach, the procedure starts by defining the first-level functional requirement, called FR0, which describes the origin of our contribution in this paper. Then, the authors set a design parameter, known as DP0, to transcribe the meaning of FR0 into the engineering words. Therefore, FR0 and DP0 are presented as follows:

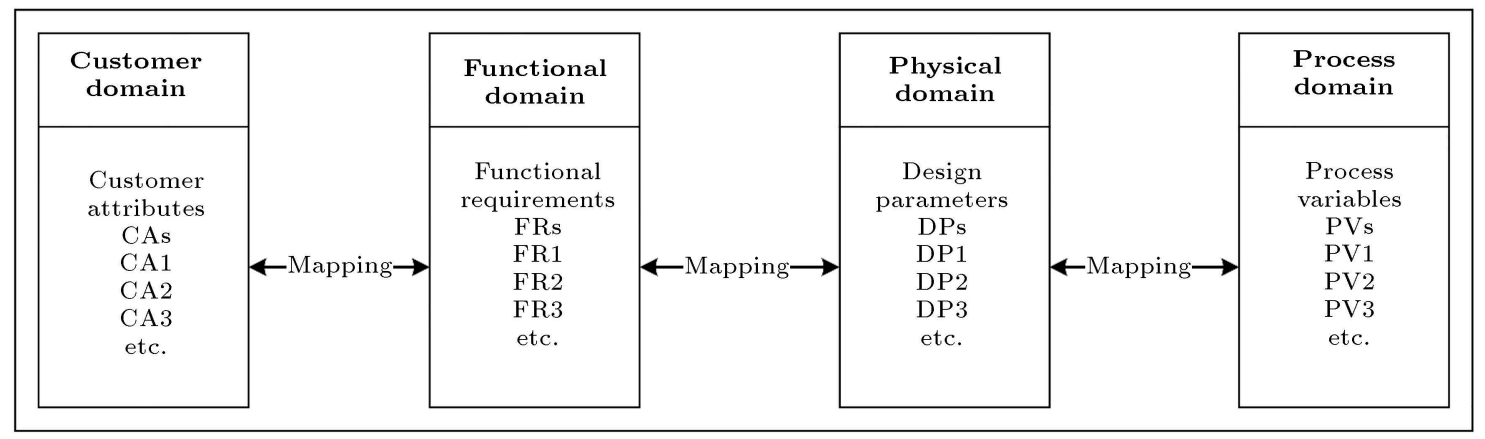

Figure 1. The axiomatic design domains. 
- FR0: A new manufacturing solution that fulfills global manufacturing requirements;

- DP0: A virtual computer integrated manufacturing architectural solution.

FR0 illustrates that the architecture of a manufacturing system is an applicable and challenging area for today's industrial worlds. The architecture is the backbone of a system with a great deal of information to satisfy the goals. However, the existence of many common drawbacks in CIM architecture and the absence of architecture for such virtual environments have been identified in the area of CIM solutions to VCIM, both for VEs and EEs. Thus, the authors proposed "A virtual computer integrated manufacturing architectural solution" as the solution or (with the axiomatic interpretation) DP0.

\subsection{Defining the second-level FRs/DPs}

At the second level of AD zigzag approach, FR0 and DP0 are broken down into detailed functional requirements that divide correspondents into the manufacturing area. Therefore, five general functional requirements are identified for the manufacturing area, whether extended or virtual, and are presented below:

- FR01: The architectural solution should be able to consider physical resources of CIM systems;

- FR02: The architectural solution should be able to address the function and process of CIM systems;

- FR03: The architectural solution should be able to consider organizational issues, rules, and command of CIM systems;

- FR04: The architectural solution should be able to consider the information flow of CIM systems;

- FR05: The architectural solution should be able to control different aspects of CIM system.

Each of these functional requirements points to one part of a manufacturing environment and describes the capabilities required for it. FR01 encounters computer integrated hardware requirements and the infrastructural necessities of the architecture to make a foundation for other aspects and future development. FR02 promises all processes and functions considered in a manufacturing system. FR03 encounters the manipulation of managerial issues in computer systems. The connection between computer integrated systems and distributed manufacturing systems involves and requires a wide field of management and business affairs. FR04 considers the requirements of data integration and information flow. The fundamental principles of effectiveness in manufacturing systems are rooted in the quality of information. FR05 emphasizes the control aspect, which is crucial for the manufacturing systems. In other words, it must utilize a number of mechanisms to control every level of the system ranging from operational to financial levels.

At this level of the AD zigzag approach, the other side of the approach is related to the secondlevel design parameters. According to the architectural layers, a one-to-one correlation can be found between the above-mentioned functional requirements. Each of the architectural layers stands for a second-level design parameter and matches its related functional requirement. The DPs are transcribed below:

- DP01: A physical layer to structure the hardware aspects;

- DP02: A functional layer to manipulate the processes and functional aspects;

- DP03: An organizational layer to administer managerial aspects;

- DP04: An informational layer to consider data and information requirements;

- DP05: A control layer to monitor the whole system.

The above DPs can be interpreted with respect to the related FRs based on the layers of the architecture. On the one hand, at the second level, functional requirements divide a manufacturing environment into five major zones; on the other hand, the five main layers are proposed in correlation with their correspondent second-level design parameters, and a wide perspective from the enterprise within these layers is expected. DP01 considers a number of hardware packages required for implementing the processes. System platform, network infrastructure, communication, and transportation are the major focuses of the physical aspects. DP02 proposes those areas that are equipped with functions and processes compatible with virtual environments. Today's extended manufacturing system consists of a variety of resources ranging from human to equipment, which operate in their own ways; therefore, modern ways are required. DP03 states that a part of the system aspects lies beyond organizational issues and describes the capabilities and responsibilities of different units and individuals. DP04 considers the information as a resource and shows the ways to meet these requirements. These ways include data centres and data processing tools. DP05 presents a number of considerations for monitoring. Since there is a need to control the operational level and shop floor tasks, high-level decisions, and long-term goals, control mechanisms are required. Mechanisms are directed at observation in terms of operation and management.

\subsection{Defining the third level FRs/DPs}

Based on the second level-design parameters, there are many functional requirements that can be categorized into the proposed five layers. Each of the layers 
necessitates some functional requirements, which are defined as below:

- FR011: A mechanism to recognize an appropriate point of automation utilization;

- FR012: A consideration regarding the virtual infrastructure for the manufacturing systems;

- FR021: Strategies to facilitate integrity and continuity in the manufacturing process;

- FR022: Methodologies to observe inter and intra relations over processes;

- FR031: Some guidelines to govern over virtual and extended resources;

- FR032: Issues to define a new manufacturing paradigm;

- FR041: Strategies to facilitate integrity and continuity in information flow;

- FR042: Issues specific to maintenance and manipulation of manufacturing data;

- FR051: A stewardship over utilization of resources and efficiency of processes;

- FR052: Infrastructural issues for a computer integrated control over manufacturing system.

The third-level FRs highly guide specific mechanisms and considerations, in which implementation tools are commonly based on promising standards. These FRs connect the architectural solution to the design parameters, forming the implementation phase. FR011 points out that the utilization of automation systems should be determined at a level appropriate for virtualization. FR012 states that there should be a foundation compatible with distributed interoperation and capable of embracing other layer hardware. FR021 considers the requirement of a seamless manufacturing flow, even in such an extended system. FR022 describes the need for methods to cover internal and external relations over the entire level of the system. FR031 and FR032 are responsible for new managerial and organizational structures and present rules to govern. FR041 explains the need for connection and stream between automation islands. FR042 considers the abilities of the enterprise in the storage of information and further exploitation of data. FR051 and FR052 state requirements for supervision over technical and tactical subjects.

The final part of AD zigzag approach is concerned with the design parameters that represent the ring between functional requirements and implementation considerations. The authors recognized the following design parameters as the third-level DPs:

- DP011: Appropriate use of automation;

- DP012: Automation as a system resource;
- DP021: Continuous flow of manufacturing;

- DP022: System overview of processes;

- DP031: Appropriate use of human and non-human resources;

- DP032: Organizational culture as a system resource;

- DP041: Continuous flow of information;

- DP042: Information as a system resource;

- DP051: Appropriate use of computers and control systems;

- DP052: Computer systems as a controller of the manufacturing system.

The third-level design parameters stand for guidance about suitable mechanisms and methodologies to ascertain our ultimate purpose. DP011 enables its related functional requirements by means of determining automation utilization. DP012 suggests promising platforms for extended manufacturing and virtualization. DP021 proposes a high-level strategy called Continuous Flow Manufacturing (CFM) that seams manufacturing processes. DP022 delineates the structure of processes and interconnection of them; to be specific, the way that they affect each other. DP031 sets the basic rules and authorities in an organizational structure for both interactions of human and non-human agents. This is a novel approach to a virtual computer integrated environment. DP032 describes culture and philosophy in association with a distributed computer integrated system and provides ways of cooperation and collaboration. DP041 seeks to establish a smooth flow of information on the basis of a computer network fed by a computerized database. The concept of Continuous Flow Information (CFI) necessitates an extensive use of computer and computer network to optimize information flow. DP042 plans to facilitate enterprises with appropriate information. It sees information as another type of resources, dispersed across the enterprise. It removes duplication, attaches separated parts, and makes a unique system for planning. DP051 indicates the performance of different aspects based on Key Performance Indicator (KPI). The financial KPIs are the most useful and financial evaluation, which are the best ways to evaluate the performance. DP052 provides a computer-based control system both for operational purpose and financial evaluation.

\section{Implementational aspects}

\subsection{Process domain elements}

To complete the AD process and reach the final conclusion on implementation considerations about the proposed architectural solution, we deal with parameters that stand for implementation. After the last 
stage of the zigzag approach and completing the lowest design parameters of $\mathrm{AD}$, there is a need for some effective tools of implementation. In the AD domains, PVs are regarded for this purpose. The final goal of these parameter values is DPs, which will be controlled through the related PVs. The authors recognized international standards as promising tools for this purpose and suggested some suitable standards for each of them. It should be noted that, according to our investigation, the proposed set of standards is expected to be one of the best methods for implementing the proposed architectural solution based on the present tools and methods. However, there are many novel ideas in this field of work to develop advanced tools and methodologies. The proposed PVs are as follows:

- PV011: ISO 23570 [78];

- PV012: ISO 15926 [79];

- PV021: ISO 14258 [80];

- PV022: ISO 15704 [81];

- PV031: ISO 11354 [82];

- PV032: ISO 11000 [83];

- PV041: ISO 15745 [84];

- PV042: ISO 15531 [85];

- PV051: IEC 62264 [86];

- PV052: ISO 22400 [87].

In the execution of physical layer, two ISO standards are suggested to command DP011 and DP012. PV011 focuses on ISO 23570 standard on distributed installation in industrial applications and specifies the detailed requirements for distributed hardware and platform. Considering the role of PV011 parameter value supporting the implementation of DP011, the required mechanisms for the appropriate use of automation are considered. The complexity of the collaboration mechanisms among the various automated devices in the shop floor-like robots, CNC machines, material handling devices, and AGVs (Automated Guided Vehicle) is at focus here, and effective procedures for their integration are provided. Moreover, with the application of ISO 23570, the process of automation trade-off design can progress better to an appropriate level of automation with respect to the consolidation considerations. This standard with its three parts provides helpful guidance on the design and selection of the physical aspects of a distributed system. Moreover, the structure of this standard provides a scalable framework for further expansion in the area of the application of automated devices and resources in VCIM systems.

For DP012, PV012 is suggested with emphasis on ISO 15926 standard of industrial automation systems for the purpose of data integration in the process.
This standard concentrates on data integration, sharing, exchange, and communication network between computer systems and resources extensively. Different resources in the manufacturing plant with their data requirements are focused on ISO 15926. This standard has different parts, which can support a wide area of manufacturing disciplines, supply chain, and life cycle stages. With respect to the information about functional requirements and physical solutions, such a standard has a high capability to select various manufacturing resources, especially automation resources. The utilization of ISO 15926 in the platform will enable automation systems as an applicable tool for distributed computer systems. By means of this $\mathrm{PV}$, the automation systems will be interpreted as a resource, which are provided and controlled by infrastructure based on ISO 15926 guidelines. ISO 15926 ensures the integration of life-cycle data manufacturing processes and, also, encompasses details of production and process operations.

In implementation details of the functional layer, the paper proposed PVs that ensure the existence of processes in a virtual network of manufacturing enterprise. For the realization of DP021, PV021 is proposed to realize the concept of continuous flow of manufacturing by means of ISO 14258. The scope of the standard specifies the set of concepts, terms, definition, and associated rules to establish a framework and a general functional method for integration of advanced process control and optimization capabilities for manufacturing systems. This international standard does not specify the enterprise-modeling process; however, it forms the basis by which enterprise-modeling standards can be developed where required. Moreover, the realization of DP022 will be performed by PV022, which focuses on ISO 15704 standard for functional capabilities provided by the enterprise resources and required to expedite business processes. The standard considers interworking of resources required to be organized and integrated into intra-relations. In addition, by means of the enterprise reference architecture, methodologies and functional support can be determined for future improvements. The scope of ISO 15704 covers the whole life of the enterprise with a wider outlook on major restructuring efforts for processes and functions.

In the third layer with a focus on the managerial dimension of architecture, the proposed architectural solution considered the definition of a new manufacturing paradigm and guidelines for governing over human resources. To meet these requirements, the solution uses the application of ISO 11354 and ISO 11000 to the related DPs. PV031 focuses on ISO 11354 to provide a framework for enterprise interoperability. ISO 11354 is particularly developed for interoperability-based solutions in IT environments, either manufacturing or service. Therefore, the standard can be applied to 
both manufacturing enterprises and service provider enterprises. It structures data, processes, and businesses in the direction of organizational vision and mission to enable interoperability among all human resource entities. A bold characteristic of ISO 11354 is related to its implementation structure, in which any tightened mechanism or a specific framework has not been specified for interoperability solution implementation. Thus, based on this structure, every human and non-human resource engaged in the enterprise processes can be managed and connected with others seamlessly. PV032 focuses on ISO 11000 for implementation consideration of DP032. ISO 11000 specifies a framework conforming to requirements of ISO 15704 , serving as a common basis to identify and coordinate standards development for modeling an enterprise. It is worth noting that ISO 11000 is not restricted to the computer integrated manufacturing field and is applicable to other types of enterprises. In addition, ISO 11000 serves as the basis for further standards of the development of models that will be computerenactable and enables business process model-based decision support leading to model-based operation, monitoring, and control.

In the fourth layer considering the informational aspects, there are many standards for integration and data exchange; however, they bring up complexities in the architecture. The paper considers the following standards that will result in a less complex system. This idea bears more relation to the current architectural aspects and enterprise integration. PV041 is presented for DP041 and focuses on ISO 15745 that ensures an appropriate approach to information exchange between applications. ISO 15745 as a standard for open systems application integration framework provides consistent information models and consistent operation models, which are the foundations of clarifying application functionality and how information is to be transmitted through the working nodes. Therefore, the standard creates a groundwork for the communication between suppliers and manufacturers to achieve a continuous flow of manufacturing. Moreover, PV042 is considered for DP042. It focuses on ISO 15531 that defines an application integration framework. This framework consists of a set of elements and rules for describing integration models and application interoperability. The integration model exploits information as a source to feed the requirement. ISO 15531 addresses the modeling of manufacturing management data, especially resources management data (resource model) and flow management data in manufacturing (flow management model). The standard is intended to facilitate information exchanges between software applications such as ERP, manufacturing management software, maintenance management software, quotation software, etc.
In the last layer, the control aspect is considered to monitor and control resource utilization and perform computer integrated control on manufacturing systems. IEC 62264 and ISO 22400 are recommended for PV051 and PV052, respectively. PV051 focuses on IEC 62264, which is an international standard for an enterprisecontrol system integration. This standard is based on ANSI/ISA-95, which has been developed for global manufacturers. It enables integration between the manufacturing operations and control domain. It aims to synchronize business strategy with operational execution in real time to enable closed-loop business control across an enterprise. It has different parts that delineate different aspects of utilization of a computer control system. Further, PV052 focuses on ISO 22400 to perform control on the manufacturing operation by means of Key Performance Indicators (KPIs). To ensure a better result of the implementation, the two parts should be performed in sequence. Based on the first part, which specifies an industry-neutral framework for defining, composing, exchanging, and using KPIs for Manufacturing Operations Management (MOM), as defined in IEC 62264, a framework for control would be created. Moreover, based on the second part, the selected number of KPIs would be provided. The KPIs are presented by means of their formula and corresponding elements (time behavior, unit/dimension, and other characteristics) to support a computer control system. A summary for the encountered PVs is presented in Table 1.

\subsection{Feasibility of the architectural solution}

To investigate the feasibility of the proposed solution, this study uses the first axiom and investigates the design matrix. As mentioned earlier in Section 4, "axiomatic design theory", to ensure the feasibility of the proposed solution, the proposed DPs and FRs should have a decoupled or uncoupled relationship. This implies that the design matrix showing the relationship among the DPs and FRs should be in the form of a diagonal or triangular matrix. To ensure the feasibility of the proposed solution at the level of the process domain, the same condition should remain for the relationship of DPs and PVs. The paper has analyzed the relationship among FRs and DPs in Figure 2 and the relationship among DPs and PVs in Figure 3 .

As the design matrixes show, the configuration of the proposed functionalities through the layers of the architecture is designed to avoid complexity. It is implied that the proposed DPs can fulfill all FRs, while their dependencies can lead to a feasible solution. Moreover, the case study considered the PVs from the domain of the ISO standards to fulfill all DPs while maintaining an acceptable level of complexity. Considering the AD theory, the implementation of 
Table 1. The explanation of the proposed standards for exploiting as PVs in the architectural solution. (The content of the table is adopted based on the international organization for standardization.)

\begin{tabular}{|c|c|c|}
\hline Standard & Exploitation & Explanation \\
\hline ISO 23570 [78] & PV011 & $\begin{array}{l}\text { Industrial automation systems and integration: Modern manufacturing tools and } \\
\text { machines are complex systems with a lot of subsystems including material handling, } \\
\text { fixturing, and transfer lines. To address and monitor these complexities in a CIM/VCIM } \\
\text { system, this standard supports the solution to deal with the interconnection of hardware } \\
\text { and physical devices. ISO } 23570 \text { specifies the interconnection of elements in the control } \\
\text { system of machine tools and similar large pieces of industrial automation. }\end{array}$ \\
\hline ISO 15926 [79] & PV012 & $\begin{array}{l}\text { Industrial automation systems and integration: This standard specifies } \\
\text { a conceptual data model at the level of plants. The specification of requirements } \\
\text { to produce, process, and transport process materials and specification of functions } \\
\text { required to produce and process, required materials are considered in this standard. } \\
\text { In addition, the selection of materials and equipment to provide the required production } \\
\text { and processing functions includes information about market available materials and } \\
\text { equipment, maintenance, and replacement of equipment. }\end{array}$ \\
\hline ISO 14258 [80] & PV021 & $\begin{array}{l}\text { Industrial automation systems: This standard provides guidelines and constraints for } \\
\text { enterprise models to model an enterprise or processes. ISO } 14258 \text { also defines concepts and } \\
\text { rules for enterprise models to help and restrict other standards that exist in the other } \\
\text { layers. This consideration is done by defining the elements to use when developing } \\
\text { an enterprise model, concepts for life-cycle phases, and how these models describe } \\
\text { hierarchy, structure, and behavior. ISO } 14258 \text { helps define the concepts and rules for } \\
\text { manufacturing systems to develop understandable models to better enable the enterprise } \\
\text { processes interoperable. }\end{array}$ \\
\hline
\end{tabular}

Industrial automation systems: ISO 15704 defines the requirements for enterprisereference architectures and methodologies and those requirements that such architectures and methodologies must satisfy. This standard is the one of the basic of enterprisereference architectures and methodologies. Enterprise-reference architectures are determined by reference methodologies and exploited technologies. By adopting a

ISO 15704 [81] PV022 reference methodology and architecture, an enterprise can cooperate and collaborate in developing and improving the enterprise utilization of resources. The cooperation and collaboration of different aspects of an enterprise reference model is defined and constrained by means of this standard. Therefore, there is a vital need in enterprise engineering and integration for a reference base and architecture to provide methodologies and technologies that can enable enterprise integration and interoperability.

Advanced automation technologies and their applications: The purpose of ISO 11354 is to specify a Framework for Enterprise Interoperability (FEI) to establish dimensions and viewpoints to address interoperability barriers, their potential solutions, and the relationships between them. This standard considers interoperability as a generic ISO 11354 [82] PV031 concept and as a necessary support to enable business collaboration. ISO 11354 assumes that common problems of interoperability are failures and the need for solutions to overcome those failures. Therefore, the standard considers enterprise interoperability to be an engineering discipline and separates it from other business concepts. ISO 11354 should be applied to manufacturing enterprises; however, it can also be applied to other kinds of enterprises. 
Table 1. The explanation of the proposed standards for exploiting as PVs in the architectural solution (The content of the table is adopted based on the international organization for standardization.) (continued).

\begin{tabular}{ll}
\hline Standard Exploitation & \multicolumn{1}{c}{ Explanation } \\
\hline & $\begin{array}{l}\text { Collaborative business relationship management: The aim of this standard is to } \\
\text { guide organizational requirements to achieve a strategic life-cycle framework and to } \\
\text { establish a collaborative communication between organizations. This standard addresses } \\
\text { both the overall requirements to establish a management system and operational process }\end{array}$ \\
ISO $11000[83] \quad$ PV032 & requirements. It specifies requirements for the effective identification, development, and \\
& management of collaborative business relationships and addresses the management \\
& system of an organization. Collaborations in the context of ISO 11000 can be multi- \\
& or single-dimensional, individual on-to-one relationships, or more frequently networked \\
& relationships involving multiple parties.
\end{tabular}

Industrial automation systems and integration: This standard defines a framework for integration including a set of elements and rules for describing integration models and application interoperability profiles. ISO 15745 is applicable to industrial automation such as discrete manufacturing, process automation, electronics assembly, and wide-area material handling. The standard develops an application integration framework and defines elements and rules that facilitate (a) systematic organization and representation of the application integration requirements using integration models and (b) development of interface specifications in the form of Application Interoperability Profiles (AIPs)

Industrial automation systems and integration: This standard aims to represent and exchange industrial manufacturing data in a computer-interpretable fashion. The objective of ISO 15531 is to provide a neutral mechanism for describing industrial manufacturing data throughout the product life-cycle. ISO 15531 specifies the characteristics for a representation of manufacturing management information over the entire industrial process. It provides the necessary mechanisms and definitions to enable manufacturing management

ISO 15531 [85] PV042 data to be shared and exchanged within the factory with other plants or companies. The standard is mainly focused on discrete manufacturing, yet not limited to it. It may also apply to any industrial processes that do not imply any contradiction or inconsistency with the basic principle of the standard. The scope of ISO 15531 includes (a) the representation of information needed to manage production and resources and (b) the exchange and sharing of production information and resources information including storing, transferring, accessing, and archiving.

Enterprise-control system integration: IEC 62264 is an international standard to enable integration throughout the control system. It is in accordance to ANSI/ISA-95 for developing automated interfaces between enterprise and control systems. This

IEC 62264 [86] PV051 standard describes the manufacturing operations management domain and the related activities, as well as transactions. The aim of the IEC 62264 is to enhance the uniformity and consistency of interface terminology and reduce the risk, cost, and errors associated with control interfaces.

Automation systems and integration: ISO 22400 focuses on KPIs for manufacturing operations management and specifies a number of KPIs as measureable and strategic index that reflect critical success factors for the organizations. The KPIs are presented by means of their formula and corresponding elements, such as time behavior, unit/dimension, and other features. ISO 22400 also provides information about where the KPIs should be used and to which groups of production they correspond. 


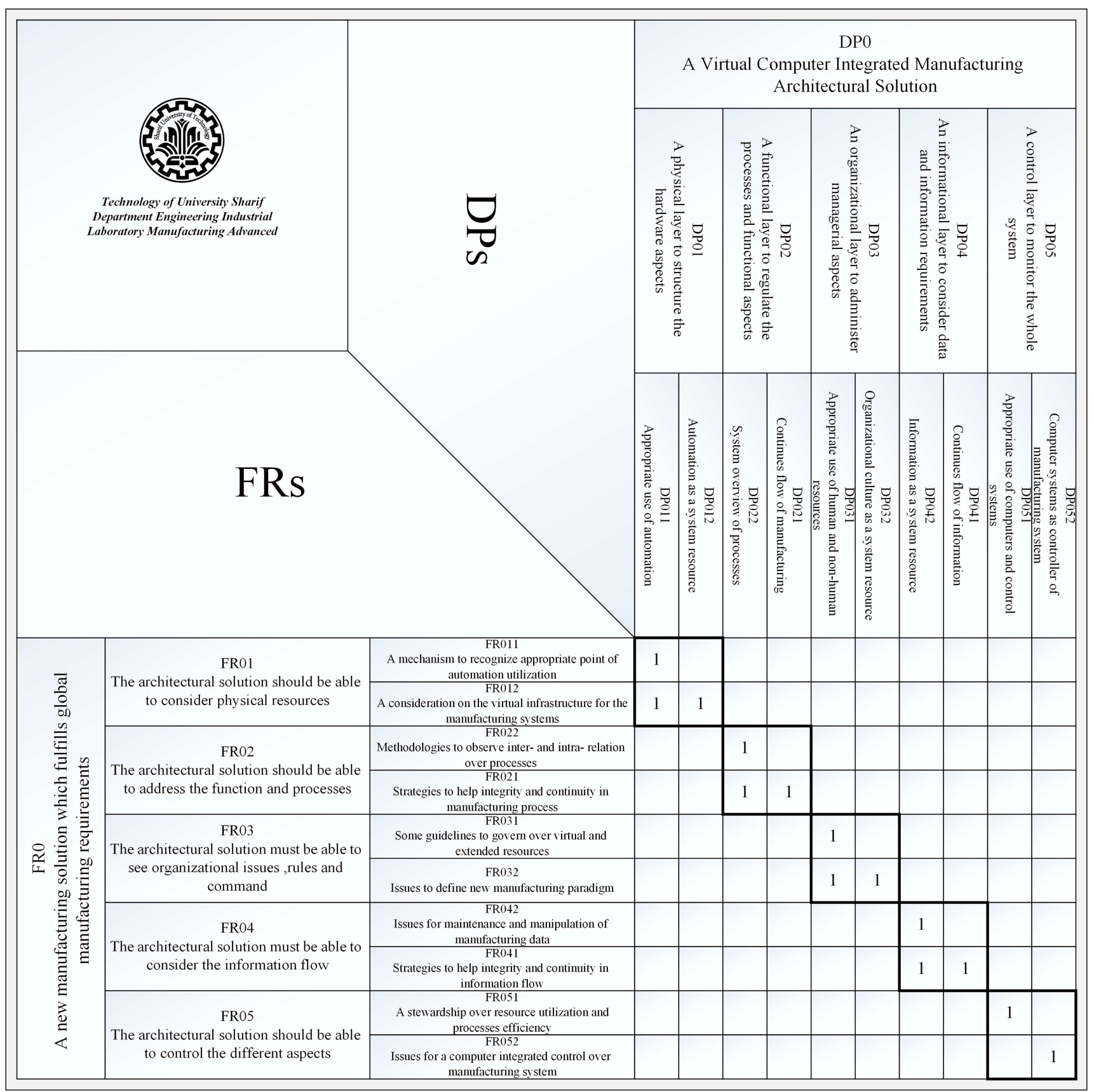

Figure 2. FR-DP design matrix.

architecture in a real process domain can be achieved by the afore-mentioned ISO standards.

\section{Discussion and conclusion}

Nowadays, the manufacturing environments are faced with the globalization paradigm that necessitates meeting a number of requirements for manufacturing environments such as concentration on customer demands, focus on increasing quality and its continuous improving trend, and shortening of the products' lifecycle. These necessities have been considered by many re- searchers and have led to different ideas in the field of effective and efficient product development and after sales services. This paper considered the capabilities of Computer Integrated Manufacturing (CIM) paradigm as a major research field for such studies. Although CIM studies have produced incredible revolutions and improvements in manufacturing systems, the complexities of globalized manufacturing environment and the inability to mitigate these complexities for addressing the different aspects of CIM system are major drawbacks found in previous pieces of research. To overcome these challenges, the Virtual CIM (VCIM) was intro- 


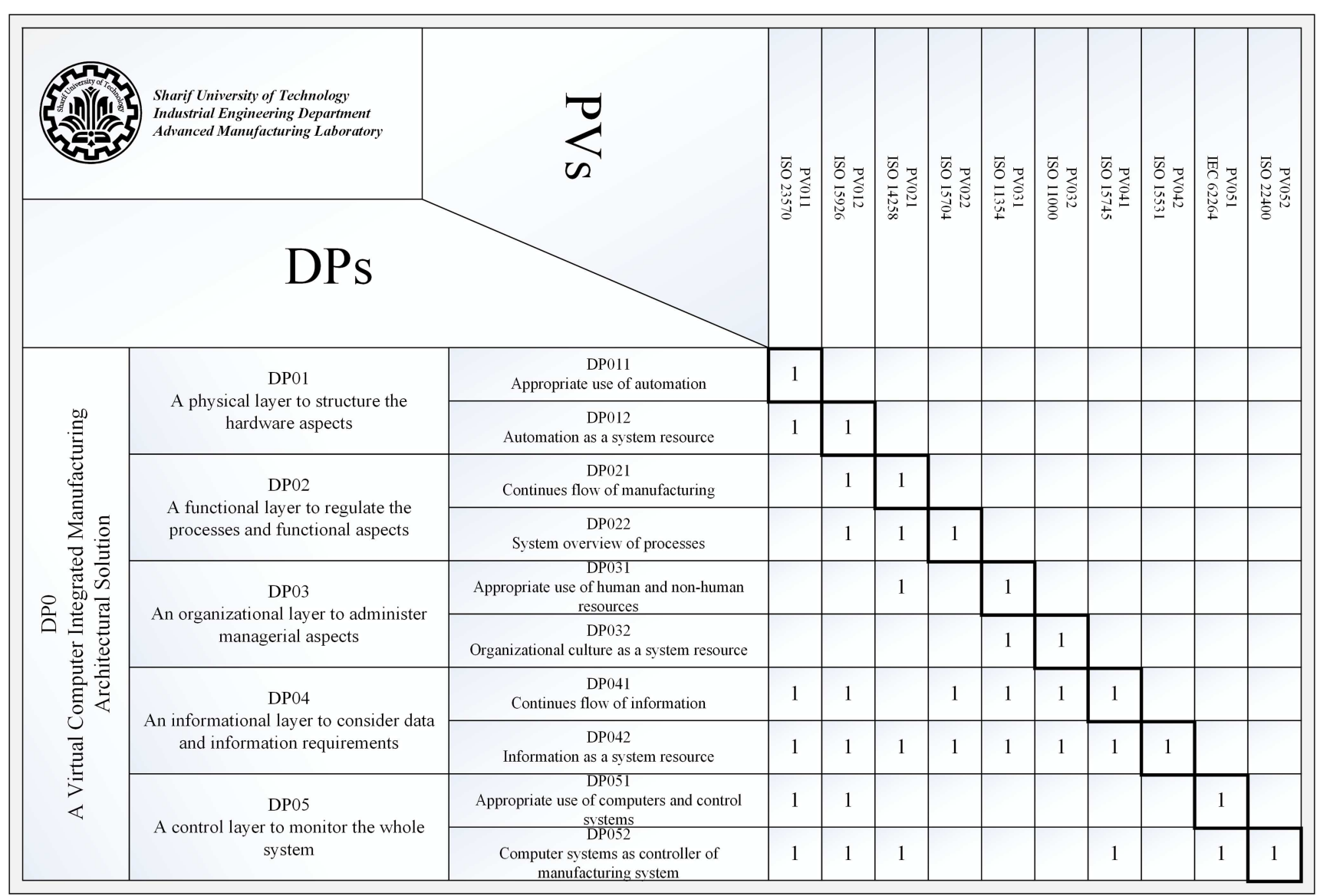

Figure 3. DP-PV design matrix.

duced as one of the most effective solutions to extend the traditional CIM solutions for the aforementioned globalized challenges. This paper studied the recent dominant pieces of research in the field of VCIM/CIM solutions, especially by considering the necessities of todays' globalized manufacturing environment. The findings suggested the major requirements and characteristics of an effective global VCIM solution in areas such as manufacturing functional capabilities and control mechanisms, human and non-human resource management issues, and manufacturing information integration. The results of the study showed the deficiency of recent researches, which were hardly capable of providing a proper solution to fully consider and handle todays' manufacturing aspects. As a result, despite all studied solutions such as agent-based theories and holonic ones, they are not providing an effective solution for designing a system.

Considering the aforementioned shortcomings, the paper proposed an effective CIM/VCIM architectural solution to cover the requirements and necessities of a global CIM/VCIM system. The core concept of the proposed architectural solution is based on a multilayer approach, which gives an extended perspective for viewing a manufacturing system within five functional areas. Each layer focuses on an operational aspect of a computer integrated system and provides a structured mechanism for its description. The dominant characteristics of the proposed layers are as follows:

- The first layer called physical layer considers the aspects related to the hardware, equipment, and human workers in a CIM/VCIM environment. These aspects transform input resources into a product by direct physical resource processing;

- The second layer called function layer describes the dynamic behavior of a manufacturing system accomplished through product development processes. The function layer results in a behavioral model in which a CIM/VCIM system consolidates its process with physical layer elements are considered;

- The third layer called organizational layer focuses on a CIM/VCIM organizational and management structure of the system. All human and non-human characteristics within the organizational roles and configurations are defined in this layer;

- The fourth layer called information layer manages the data and information aspects of a CIM/VCIM system. These aspects can include data and information requirements as manufacturing processes' input and resulted data and information from those 
processes. Moreover, the information flow of the manufacturing system is organized in this layer;

- The fifth layer called control layer considers the whole supportive processes such as monitoring and control, inspection, and decision-making in a CIM/VCIM system. This layer monitors the whole system processes and covers the indirect support and supervision of necessary tasks in a manufacturing system.

Since the complexity of the solution in this area is too high, the paper applied Axiomatic Design (AD) Theory to ensure the feasibility of its proposed solution. This theory was used for both ensuring the viability of the proposed architectural solution and, also, verifying the feasibility of the implementational aspects. The aspects related to implementation of the solution were considered by the ISO standards. The results achieved by $\mathrm{AD}$ axioms approve the feasibility of the proposed solution for manufacturing systems and their implementational aspects. As for suggestions for further study, the authors propose exploiting other innovative approaches, such as TRIZ, to develop other architectural solutions, especially for other businesses and non-manufacturing systems.

\section{Acknowledgement}

The authors gratefully appreciate Professor Mahmoud Houshmand and Professor Gholamhossein Farrahi for their invaluable advice and comments, which improved and enhanced our research.

\section{References}

1. Valilai, O.F. and Houshmand, M. "A collaborative and integrated platform to support distributed manufacturing system using a service-oriented approach based on cloud computing paradigm", Rob. and Com. Integ. Man., 29(1), pp. 110-127 (2013).

2. Koren, Y., The Global Manufacturing Revolution: Product-Process-Business Integration and Reconfigurable Systems, John Wiley \& Sons, UK (2010).

3. Zhang, W. and Xie, S. "Agent technology for collaborative process planning: a review", The Int. J. of Adv. Man. Tech., 32(3), pp. 315-325 (2007).

4. Nagalingam, S.V. and Lin, G.C. "CIM-still the solution for manufacturing industry", Rob. and Com. Integ. Man., 24(3), pp. 332-344 (2008).

5. Houshmand, M. and Valilai, O.F. "LAYMOD: a layered and modular platform for CAx product data integration based on the modular architecture of the standard for exchange of product data", Int. J. of Com. Integ. Man., 25(6), pp. 473-487 (2012).

6. Valilai, O.F. and Houshmand, M. "INFELT STEP: An integrated and interoperable platform for collaborative
CAD/CAPP/CAM/CNC machining systems based on STEP standard", Int. J. of Com. Integ. Man., 23(12), pp. 1095-1117 (2010).

7. Wang, X.V. and Xu, X.W. "An interoperable solution for cloud manufacturing", Rob. and Com. Integ. Man., 29(4), pp. 232-247 (2013).

8. Li, Q. "Applications integration in a hybrid cloud computing environment: modelling and platform", Ent. Info. Sys., 7(3), pp. 237-271 (2013).

9. Valilai, O.F. and Houshmand, M. "A platform for optimisation in distributed manufacturing enterprises based on cloud manufacturing paradigm", Int. J. of Com. Integ. Man., 27(11), pp. 1031-1054 (2014).

10. Houshmand, M. and Valilai, O.F. "A layered and modular platform to enable distributed CAx collaboration and support product data integration based on STEP standard", Int. J. of Com. Integ. Man., 26(8), pp. 731750 (2013).

11. Zhou, N. "Development of an agent based VCIM resource scheduling process for small and medium enterprises", Int. Ass. of Eng., 25(2), pp. 31-50 (2010).

12. Nagalingam, S.V. and Lin, G. "Latest developments in CIM", Rob. and Com. Integ. Man., 15(6), pp. 423-430 (1999).

13. Wang, D., Nagalingam, S., and Lin, G. "Development of an agent-based virtual CIM architecture for small to medium manufacturers", Rob. and Com. Integ. Man., 23, pp. 1-16 (2007).

14. Zhou, N., Nagalingam, S., and Lin, G. "Application of virtual CIM in small and medium manufacturing enterprises", Int. J. of Com. Integ. Man., 25(12), pp. 131-154 (2011).

15. Delaram, J. and Valilai, O.F. "Development of a novel solution to enable integration and interoperability for cloud manufacturing", Procedia CIRP, 52, pp. 6-11 (2016).

16. Scheer, A.W., CIM Computer Integrated Manufacturing: Computer Steered Industry, Springer Publishing Company (2012).

17. Rehg, J.A. and Kraebber, H.W., Computer-Integrated Manufacturing, Prentice Hall (2005).

18. McGaughey, R.E. and Roach, D. "Obstacles to computer integrated manufacturing success: a study of practitioner perceptions", Int. J. of Com. Integ. Man., 10(1), pp. 256-265 (1997).

19. Delaram, J. and Valilai, O.F. "A novel solution for manufacturing interoperability fulfillment using interoperability service providers", Procedia CIRP, 63, pp. 774-779 (2017).

20. Zhou, N. "Inside virtual CIM", Intel. Cont. and Com. Eng., 11(5), pp. 163-175 (2011).

21. Mitchell Jr, F., CIM Systems: An Introduction to Computer-Integrated Manufacturing, Prentice-Hall (1991) . 
22. Groover, M.P., Automation, Production Systems, and Computer-Integrated Manufacturing, PrenticeHall (2007).

23. Wang, X., Wong, T., and Wang, G. "An ontological intelligent agent platform to establish an ecological virtual enterprise", Exp. Sys. with App., 39(8), pp. 7050-7061 (2012).

24. Nagalingam, S.V. and Lin, G. "A unified approach towards CIM justification", Com. Integ. Man. Sys., 10(2), pp. 133-145 (1997).

25. Zhou, N. "Virtual CIM", Intel. Cont. and Com. Eng., 21(9), pp. 31-40 (2015).

26. Valilai, O.F. and Houshmand, M. "Depicting additive manufacturing from a global perspective; using cloud manufacturing paradigm for integration and collaboration", Pro. of the Inst. of Mech. Eng., Part B: J. of Eng. Man., 229(12), pp. 2216-2237 (2014).

27. Erenay, O., Hashemipour, M., and Kayaligil, S. "Virtual reality in requirement analysis for CIM system development suitable for SMEs", Int. J. of Pro. Res., 40(15), pp. 3693-3708 (2002).

28. Nagalingam, S., Lin, G., and Wang, D. "Resource scheduling for a virtual CIM system", Adv. Man., 10(2), pp. 269-294 (2007).

29. Zhou, N., Nagalingam, S., and Lin, G. "Application of virtual CIM in small and medium manufacturing enterprises", Int. J. of Pro. Res., 9(4), pp. 161-164 (2007).

30. Browne, J., The Extended Enterprise-Manufacturing and the Value Chain, Springer (1995).

31. Jagdev, H. and Browne, J. "The extended enterprisea context for manufacturing", Pro. Plan. and Cont., 9(3), pp. 216-229 (1998).

32. Martinez, M.T. "Virtual enterprise-organisation, evolution and control", Int. J. of Pro. Eco., 74(1), pp. 225-238 (2001).

33. Park, K.H. and Favrel, J. "Virtual enterpriseinformation system and networking solution", Com. and Ind. Eng., 37(1), pp. 441-444 (1999).

34. Liu, N., Li, X., and Shen, W. "Multi-granularity resource virtualization and sharing strategies in cloud manufacturing", J. of Net. and Com. App., 46, pp. $72-82$ (2014).

35. Morariu, O., Borangiu, T., and Raileanu, S. "vMES: Virtualization aware manufacturing execution system", Comp. in Ind., 67(2), pp. 27-37 (2015).

36. Buyya, R., Vecchiola, C., and Selvi, S.T. "Virtualization, in mastering cloud computing", Comp. in Ind., 67(2), pp. 71-109 (2013).

37. Van Geenhuizen, M. and Nijkamp, P. "Knowledge virtualization and local connectedness among young globalized high-tech companies", Tech. Fore. and Soc. Chan., 79(7), pp. 1179-1191 (2012).

38. Camarinha-Matos, L.M. and Afsarmanesh, H. "A comprehensive modeling framework for collaborative networked organizations", J. of Intel. Man., 18(5), pp. 529-542 (2007).
39. Brown, E.A. "Reinventing government research and development: A status report on management initiatives and reinvention efforts at the Army Research Laboratory", J. of Net. and Com. App., 62(1), pp. 78-111 (1998).

40. Vassiliou, M. "The virtual research laboratory: taxonomy and analysis. in aerospace", Com. and Ind. Eng., 37(1), pp. 441-444 (1999).

41. Narula, R. "R\&D collaboration by SMEs: new opportunities and limitations in the face of globalisation", Techno., 24(2), pp. 153-161 (2004).

42. Ross, J.W., Weill, P., and Robertson, D. "Enterprise architecture as strategy: Creating a foundation for business execution", Har. Bus. Rev., 65(3), pp. 34-44 (2006).

43. Browne, J. and Zhang, J. "Extended and virtual enterprises-similarities and differences", Int. J. of $\mathrm{Ag}$. Man. Sys., 1(1), pp. 30-36 (1999).

44. Zhang, J., Chan, F., and Li, P. "Agent-and CORBAbased application integration platform for an agile manufacturing environment", The Int. J. of Adv. Man. Tech., 21(6), pp. 460-468 (2003).

45. Huang, B. "A framework for virtual enterprise control with the holonic manufacturing paradigm", Com. In Ind., 49(3), pp. 299-310 (2002).

46. Odrey, N.G. and Mejí, G. "A re-configurable multiagent system architecture for error recovery in production systems", Rob. and Com. Integ. Man., 19(1), pp. 35-43 (2003).

47. Hernandez-Matias, J. "An integrated modelling framework to support manufacturing system diagnosis for continuous improvement", Rob. and Com. Iinteg. Man., 24(2), pp. 187-199 (2008).

48. Lin, C.P. and Jeng, M. "An expanded SEMATECH CIM framework for heterogeneous applications integration", Man. and Cyber., 36(1), pp. 76-90 (2006).

49. Nahm, Y.E. and Ishikawa, H. "A hybrid multi-agent system architecture for enterprise integration using computer networks", Rob. and Com. Integ. Man., 21(3), pp. 217-234 (2005).

50. Williamson, A. and Deasley, P. "Systems thinking and computer-integrated manufacturing", Sys. Prac Tech., 7(1), pp. 9-23 (1994).

51. Trappey, A.J., Liu, T.H., and Hwang, C.T. "Using EXPRESS data modeling technique for PCB assembly analysis", Com. In Ind., 34(1), pp. 111-123 (1997).

52. Delaram, J. and Valilai, O.F. "An architectural view to computer integrated manufacturing systems based on axiomatic design theory", Com. In Ind., 100, pp. 96-114 (2018).

53. Suh, N.P., The Principles of Design, Oxford University Press, UK (1990).

54. Kulak, O. and Kahraman, C. "Fuzzy multi-attribute selection among transportation companies using axiomatic design and analytic hierarchy process", Info. Sci., 170(2), pp. 191-210 (2005). 
55. Carnevalli, J.A., Miguel, P.A.C., and Calarge, F.A. "Axiomatic design application for minimising the difficulties of QFD usage", Int. J. of Pro. Eco., 125(1), pp. 1-12 (2010).

56. Peck, J., Nightingale, D., and Kim, S.G. "Axiomatic approach for efficient healthcare system design and optimization", CIRP Ann. Man. Tech., 59(1), pp. 469$472(2010)$.

57. Linke, B.S. and Dornfeld, D.A. "Application of axiomatic design principles to identify more sustainable strategies for grinding", J. of Man. Sys., 52(4), pp. 49-72 (2012).

58. Cochran, D.S. "The application of axiomatic design and lean management principles in the scope of production system segmentation", Int. J. of Pro. Res., 38(6), pp. 1377-1396 (2000).

59. Valilai, O.F. and Houshmand, M. "A manufacturing ontology model to enable data integration services in cloud manufacturing using axiomatic design theory", in Cloud-Based Design and Manufacturing (CBDM): A Service-Oriented Product Development Paradigm for the 21st Century, Springer (2014).

60. Suh, N.P., Axiomatic Design: Advances and Applications, Oxford University Press, UK (2001).

61. Kim, S.J., Suh, N.P., and Kim, S.G. "Design of software systems based on axiomatic design", Rob. and Com. Integ. Man., 8(4), pp. 243-255 (1991).

62. Rechtin, E., Systems Architecting: Creating and Building Complex Systems, Prentice-Hall (2001).

63. Albano, L.D. and Suh, N.P. "Axiomatic design and concurrent engineering", Com. Aid. Des., 26(7), pp. 499-504 (1994).

64. Bae, S., Lee, J.M., and Chu, C.N. "Axiomatic design of automotive suspension systems", CIRP Ann. Man. Tech., 51(1), pp. 115-118 (2002).

65. Suh, N.P. "Designing-in of quality through axiomatic design", Rel, Trans., 44(2), pp. 256-264 (1995).

66. Gonçalves-Coelho, A.M. and Mourao, A.J. "Axiomatic design as support for decision-making in a design for manufacturing context: A case study", Int. J. of Pro. Eco., 109(1), pp. 81-89 (2007).

67. Cebi, S. and Kahraman, C. "Indicator design for $p$ passenger car using fuzzy axiomatic design principles", Exp. Sys. with App., 37(9), pp. 6470-6481 (2010).

68. Suh, N.P. "Axiomatic design of mechanical systems", J. of Mech. Des., 117(B), pp. 2-10 (1995).

69. Gebala, D.A. and Suh, N.P. "An application of axiomatic design", Res. in Eng. Des., 3(3), pp. 149-162 (1992).

70. Togay, C., Dogru, A.H., and Tanik, J.U. "Systematic component-oriented development with axiomatic design", J. of Sys. and Soft., 81(11), pp. 1803-1815 (2008).

71. Ferrer, I. "Methodology for capturing and formalizing DFM Knowledge", Rob. and Com. Integ. Man., 26(5), pp. $420-429$ (2010).
72. Cebi, S., Celik, M., and Kahraman, C. "Structuring ship design project approval mechanism towards installation of operator-system interfaces via fuzzy axiomatic design principles", Info. Sci., 180(6), pp. 886-895 (2010).

73. Heo, G. and Lee, S.K. "Design evaluation of emergency core cooling systems using axiomatic design", Nuc. Eng. and Des., 237(1), pp. 38-46 (2007).

74. Thielman, J. "Evaluation and optimization of General Atomics' GT-MHR reactor cavity cooling system using an axiomatic design approach", Nuc. Eng. and Des., 235(13), pp. 1389-1402 (2005).

75. Yi, J.W. and Park, G.J. "Development of a design system for EPS cushioning package of a monitor using axiomatic design", Adv. in Eng. Soft., 36(4), pp. 273284 (2005).

76. Hirani, H. and Suh, N.P. "Bearing design using multiobjective genetic algorithm and axiomatic design approaches", Tri. Int., 38(5), pp. 481-491 (2005).

77. Janthong, N., Brissaud, D., and Butdee, S. "Combining axiomatic design and case-based reasoning in an innovative design methodology of mechatronics products", CIRP J. of Man. Sci. and Tech., 2(4), pp. 226-239 (2010).

78. http://www.iso.org/iso/iso_catalogue/catalogue_tc/ catalogue_detail.htm?csnumber $=39926$.

79. http://www.iso.org/iso/iso_catalogue/catalogue_tc/ catalogue_detail.htm?csnumber $=29556$.

80. http://www.iso.org/iso/iso_catalogue/catalogue_tc/ catalogue_detail.htm?csnumber $=24020$.

81. http://www.iso.org/iso/iso_catalogue/catalogue_tc/ catalogue_detail.htm?csnumber $=28777$.

82. http://www.iso.org/iso/iso_catalogue/catalogue_tc/ catalogue_detail.htm?csnumber $=50417$.

83. http://www.iso.org/iso/catalogue_detail.htm? csnumber $=46559$.

84. http://www.iso.org/iso/iso_catalogue/catalogue_tc/ catalogue_detail.htm?csnumber $=30418$.

85. http://www.iso.org/iso/iso_catalogue/catalogue_tc/ catalogue_detail.htm?csnumber $=31583$.

86. http://www.iso.org/iso/catalogue_detail.htm? csnumber $=57308$.

87. http://www.iso.org/iso/catalogue_detail.htm? csnumber $=54497$.

\section{Biographies}

Jalal Delaram is a PhD student at the Department of Industrial Engineering in Sharif University of Technology (SUT), Tehran, Iran. Currently, he is conducting his research on cloud manufacturing and the next generation of logistic services named 5PL in Advanced Manufacturing Laboratory (AML). He has earned his BSc and MSc degrees in Industrial Engineering at SUT. His major fields of research are cloud manufacturing, 
Computer Integrated Manufacturing (CIM), and Manufacturing Operations Management (MOM).

Omid Fatahi Valilai holds a $\mathrm{PhD}$ at the Industrial Engineering Department, Sharif University of Technology (SUT), Tehran, Iran. He is now as Assistant
Professor at the Department of Industrial Engineering at Sharif University of Technology, Tehran, Iran. He is also the Vice President of Graduate Studies at the Department of Industrial Engineering. His major research interests include Computer Integrated Manufacturing (CIM), especially cloud manufacturing. 